The Astrophysical Journal, 216:659-664, 1977 September 1

(C) 1977. The American Astronomical Society. All rights reserved. Printed in U.S.A.

\title{
CONFIRMATION OF THE PRESENCE OF IRON HYDRIDE IN SUNSPOTS AND COOL STARS
}

\author{
ROBERT F. WING \\ Astronomy Department, Ohio State University \\ AND \\ Judith Cohen and James W. BRault \\ Kitt Peak National Observatory* \\ Received 1976 December 17; accepted 1977 March 7
}

\begin{abstract}
A high-resolution sunspot spectrum clearly shows the presence of the $9896 \AA$ and $8691 \AA$ bands of iron hydride $(\mathrm{FeH})$. At least 332 lines of the two bands, accounting for $80 \%$ of the lines registered in the laboratory, can be identified with certainty in the spot spectrum; most of these are unblended. Our estimate that the $9896 \AA$ band is $2.0 \pm 0.2$ times stronger than the $8691 \AA$ band supports the idea, suggested earlier by laboratory deuteride experiments, that the $9896 \AA$ band is the $(0,0)$ transition. No evidence for $\mathrm{FeH}$ is found in the spectrum of the solar disk.

Image-tube spectrograms of $M$ dwarfs and $S$ stars have been taken in the near-infrared at $86 \AA \mathrm{mm}^{-1}$ to examine the feature which Nordh, Lindgren, and Wing have proposed is due to the $9896 \AA$ band of $\mathrm{FeH}$. At this dispersion enough band structure is evident to confirm the identification in both kinds of stars.
\end{abstract}

Subject headings: line identifications - molecular processes — stars: late-type — Sun: spectra Sun: sunspots

\section{INTRODUCTION}

Despite the well-established presence of several other metallic hydrides in the Sun and cool stars, no identification of iron hydride $(\mathrm{FeH})$ was made prior to the work of Carroll and McCormack (1972), who provided the first published data on the laboratory spectrum of this molecule and reported a large number of coincidences with weak solar lines in the blue and green spectral regions. Although a fuller report has now been published (Carroll, McCormack, and O'Connor 1976), the identification of the blue and green bands of $\mathrm{FeH}$ still rests largely upon statistical evidence since these regions of the solar spectrum are so cluttered with atomic lines and many of the weak lines in the same regions remain unidentified.

Laboratory measurements of the $8691 \AA$ band of $\mathrm{FeH}$ have recently been published by McCormack and O'Connor (1976); this band was not, however, found in the solar spectrum. An additional FeH band at $9896 \AA$ has been studied in the laboratory by Klynning and Lindgren (1973), but they likewise were unable to find evidence of it in the solar spectrum.

The failure to identify the infrared $\mathrm{FeH}$ bands in the Sun is less disturbing when we recall that the available solar atlases in the near-infrared refer to light from the disk, while the $\mathrm{FeH}$ bands would be expected to be stronger in sunspot spectra. Indeed,

* Operated by the Association of Universities for Research in Astronomy, Inc., under contract with the National Science Foundation.
Carroll, McCormack, and O'Connor (1976) found that their statistical evidence for the blue and green bands of $\mathrm{FeH}$ was strongest when they limited consideration to solar lines that are enhanced in spot spectra or seen only in spots. Clearly, if the identifications of the blue and green bands are correct, the $\mathrm{FeH}$ bands in the much cleaner infrared spectrum should be easily visible in spot spectra of sufficient resolution. Such a spectrum is now available, and our study of it is discussed in $\S$ II.

Nordh, Lindgren, and Wing (1977) have recently proposed that the $9896 \AA$ band of $\mathrm{FeH}$ is responsible not only for a feature centered at $9910 \AA$ found by Wing and Ford (1969) in the spectra of late M dwarfs but also for a band noted by Wing (1972) in the spectra of certain cool stars of type S. They found that the shape of the band in a photoelectric scan of an $S$ star agreed with the degraded laboratory spectrum, but the available observations of $\mathbf{M}$ dwarfs had too low resolution to show any structure. We feel that it is important to remove the remaining uncertainty in the identification of the Wing-Ford band in $M$ dwarfs, not only because of its potential value in studies of the atmospheres of these stars but also because of its use as an indicator of the stellar content of external galaxies (Whitford 1977). It is also important, from the viewpoint of atmospheric structure studies, to know if it is the same band that is so greatly enhanced in both $\mathbf{M}$ dwarfs and $\mathrm{S}$-type giants, relative to $\mathbf{M}$ giants. In $\S$ III we present spectroscopic observations of sufficient resolution to confirm the identification of $\mathrm{FeH}$ in both kinds of stars. 


\section{IRON HYDRIDE IN SUNSPOTS}

\section{a) The FTS Observation}

A new Fourier-transform spectrometer (FTS) designed by Brault has recently been put into operation at the McMath solar telescope of Kitt Peak National Observatory (KPNO). This instrument has a $1 \mathrm{~m}$ path for the movable mirrors and is capable of resolutions up to $0.005 \mathrm{~cm}^{-1}$.

On 1976 March 31, during the instrument testing phase, a 20 min observation of a sunspot was recorded. The entrance aperture used, a circle $9^{\prime \prime}$ in diameter, was smaller than the umbra of the spot, but the seeing was not particularly good and some penumbral light probably entered the instrument. The observation was made through 1.22 air masses on a dry day, and atmospheric absorption lines in the spectrum are unusually weak. The spectrum has excellent quality from $6000 \AA$ to $10000 \AA$ and is usable from $4900 \AA$ to $10900 \AA$; this wavelength range is limited at the short-wavelength end by the rapidly decreasing light level and GG 475 filter, and at the long-wavelength end by the response of the silicon diode.

\section{b) The 9896 A Band}

The lines of this band extend from $9896.2 \AA$ longward to at least $10205 \AA$ (Klynning and Lindgren 1973). The band has an open, "many-lined" structure, typical of hydrides. The greatest line density occurs in the region from roughly $9900 \AA$ to $9930 \AA$, close to the short-wavelength limit of the band, but there is no actual band head in the sense of a strong concentration of lines at this limit. A secondary maximum in the band intensity occurs at $10050-10065 \AA$ where there is a group of strong lines. Attempts to analyze the band, or even to arrange its lines into branches, have not been successful.

The FTS sunspot spectrum shows a great many lines between about $9900 \AA$ and $10200 \AA$ that do not appear in a spectrum of the center of the solar disk obtained with similar resolution, or in the disk spectrum published by Delbouille and Roland (1963). A comparison with the laboratory line list of Klynning and Lindgren (1973) showed at once that the overwhelming majority of these lines belong to the $9896 \AA$ band of $\mathrm{FeH}$. The correspondence between laboratory and sunspot spectra is particularly good in the interval $9896-10000 \AA$, in which we consider 83 of the 87 lines measured in the laboratory to be definitely present in the spot, with three others probably present; the one missing line is also present if we accept an error of $0.7 \AA$ in the laboratory wavelength. In the interval 10000-10205 $\AA$ the lines are weaker and the correspondence is less good, but 72 of the 87 laboratory lines are judged to be definitely present in the spot, with seven more probably present. Most of the missing lines in this interval are the weaker components of blends which are only marginally visible on the laboratory tracing. Altogether 155 lines of the $9896 \AA$ band, or $89 \%$ of the lines listed by Klynning and Lindgren, are certainly present in the sunspot spectrum, while an additional 10 lines $(6 \%)$ are probably present.

Contamination of the solar spectrum by atomic lines, $\mathrm{CN}$ lines, or atmospheric $\mathrm{H}_{2} \mathrm{O}$ lines is very slight near $1 \mu \mathrm{m}$, and the sunspot spectrum from $9900 \AA$ to $10100 \AA$ comes close to being a pure FeH spectrum. The remaining unidentified lines in this region are for the most part weaker than the known $\mathrm{FeH}$ lines, and it seems likely that many of them are

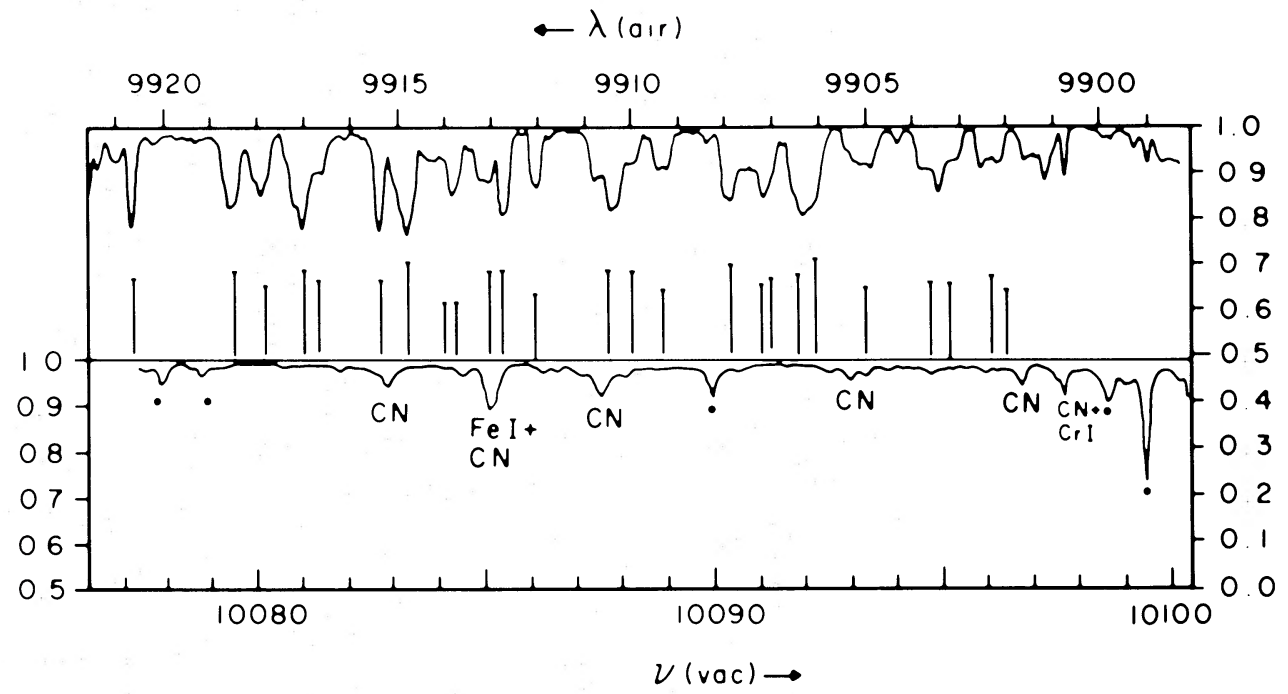

FIG. 1. - The spectrum of a sunspot (above) is compared to the spectrum of the center of the solar disk (below) in the region of maximum $\mathrm{FeH}$ absorption in the $9896 \AA$ band. The intensity scale at the left applies to the disk spectrum, the one at the right to the spot spectrum. Vacuum wavenumber $\left(\right.$ in $\mathrm{cm}^{-1}$ ) is indicated at the bottom, wavelength in air (in $\AA$ ) at the top. Vertical bars indicate the positions and relative strengths of $\mathrm{FeH}$ lines observed by Klynning and Lindgren in the laboratory. The features seen in the disk spectrum can be identified with atmospheric water lines (dots) or solar lines of $\mathrm{CN}$ and neutral atoms; by contrast, most of the absorption in the spot spectrum can be attributed to $\mathrm{FeH}$. 
$\mathrm{FeH}$ lines that have not yet been measured in the laboratory. Longward of $10100 \AA$ the FeH lines are less dominant and the spot spectrum contains a large number of unidentified lines that are probably not due to $\mathrm{FeH}$.

Figure 1 shows the sunspot spectrum from $9900 \AA$ to $9920 \AA$, the region of maximum $\mathrm{FeH}$ absorption which, according to Nordh, Lindgren, and Wing (1977), provides the feature that is seen at very low resolution in the spectra of cool $\mathbf{M}$ dwarfs. Below it is an FTS spectrum of the center of the solar disk, drawn to the same scale but displaced vertically. The horizontal scale is proportional to wavenumber, so that wavelength increases to the left on a slightly nonlinear scale. Vertical bars have been drawn at the positions of the $\mathrm{FeH}$ lines measured by Klynning and Lindgren (1973), and their lengths are proportional to their intensities, which we have taken from the published tracing of the $\mathrm{FeH}$ emission spectrum.

It is clear that nearly all the absorption in the sunspot spectrum of Figure 1 is due to $\mathrm{FeH}$. The laboratory and sunspot lines are well correlated in intensity as well as in wavelength. It is also noteworthy that the disk spectrum shows no clear evidence of even the strongest $\mathrm{FeH}$ lines. Although the region shown contains the greatest concentration of $\mathrm{FeH}$ lines, we wish to emphasize that convincing identifications of $\mathrm{FeH}$ lines in the spot spectrum can be made throughout the interval 9896-10200 $\AA$, a region 15 times wider than the one shown in Figure 1.

\section{c) The 8691 A Band}

This $\mathrm{FeH}$ band, measured by McCormack and O'Connor (1976), also has an open structure that has defied rotational analysis. Its lines extend from $8690.8 \AA$ to beyond $8900 \AA$, with their maximum concentration within about $40 \AA$ of the short-wavelength limit.
The identification of $\mathrm{FeH}$ lines in the spot spectrum has been almost as straightforward in this band as in the $9896 \AA$ band. The lines are on the average somewhat weaker, but again the majority of the lines in the spot spectrum are clearly due to $\mathrm{FeH}$. Of the 239 lines in the laboratory tabulation, we consider 177 (or $74 \%$ ) to be certainly present in the spot spectrum, and most of the remaining lines are masked or blended.

Figure 2 shows a segment of the $8691 \AA$ band from $8694 \AA$ to $8711 \AA$, in the region of maximum line concentration near the short-wavelength limit. As in Figure 1, the spot and disk spectra are compared, and vertical bars indicate the positions and intensities of $\mathrm{FeH}$ lines measured in the laboratory. Again, nearly all the absorption in the spot spectrum can be attributed to $\mathrm{FeH}$.

\section{d) Relative Strengths of the Infrared Bands}

It has not previously been possible to compare the intensities of the $9896 \AA$ and $8691 \AA$ bands because they have been observed in different laboratories. There is, however, some laboratory evidence that the $9896 \AA$ band is the $(0,0)$ transition and, as such, is likely to be the stronger of the two. This evidence consists of the small shift that is observed at the $9896 \AA$ band when the hydrogen in the King furnace is replaced with deuterium. The isotope shift for the $8691 \AA$ band is considerably greater (Carroll, McCormack, and O'Connor 1976). The stellar evidence (Nordh, Lindgren, and Wing 1977) is consistent with the idea that the $9896 \AA$ band is the stronger one, but it has not been possible to measure the $8691 \AA$ band quantitatively in stars because it is masked by $\mathrm{TiO}$ in late $\mathrm{M}$ stars and by one of the unidentified Keenan bands in $\mathrm{S}$ stars.

The sunspot spectrum discussed here is the first spectrum, to our knowledge, to show both $\mathrm{FeH}$ bands clearly. Since the bands have not been analyzed, it is

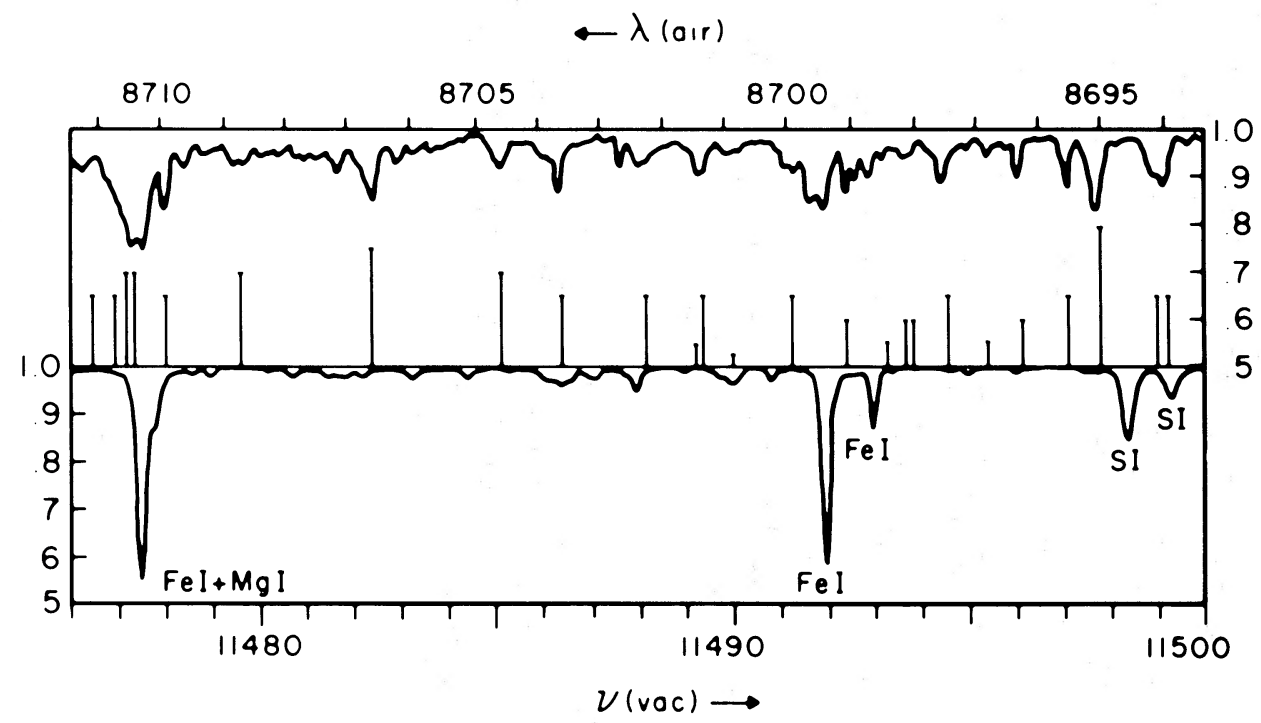

FIG. 2.- Spectra of a sunspot (above) and the solar disk (below) in the region of maximum absorption in the $8691 \AA$ band. Bars represent laboratory measurements of FeH lines by McCormack and O'Connor. See caption to Fig. 1. 
not possible to compare corresponding lines in the two bands or to correct their strengths for possible differences in excitation. The best that we can do is to measure the absorption averaged over corresponding portions of the two bands. For this purpose we have selected $10 \AA$ intervals close to the short-wavelength limits of the bands: $8695-8705 \AA$ and $9905-9915 \AA$. Both intervals have high $\mathrm{FeH}$ line densities and little contamination in the sunspot spectrum; both are included in the regions shown in Figures 1 and 2 . We define the mean absorption as the fraction of the light in the chosen spectral interval that is absorbed by $\mathrm{FeH}$, and we have assumed that in these intervals all the absorption is due to $\mathrm{FeH}$ except for a few lines that clearly are not. The results are $7.4 \%$ for the region in the $9896 \AA$ band and $3.7 \%$ for the region in the $8691 \AA$ band.

If we assume that we have indeed chosen "corresponding" regions in the two bands in the sense that they include lines having the same quantum numbers, and if the two bands have the same lower vibrational level, then the ratio of the mean absorptions obtained above, 2.0, may be interpreted as the ratio of the intensity of the $9896 \AA$ band to that of the $8691 \AA$ band. Apart from possible errors in our assumptions, the greatest source of uncertainty in this ratio is in the placement of the continuum; possible departures from the continua used in the calculation could change the ratio by \pm 0.2 . In any event, the result that the $9896 \AA$ band is about twice as strong as the $8691 \AA$ band is consistent with the impression gained from simple inspection of Figures 1 and 2. This result, coupled with the evidence from deuteride experiments, makes it virtually certain that the $9896 \AA$ band is the $(0,0)$ transition. In that case the most likely assignment for the $8691 \AA$ band is the $(1,0)$ transition, as Carroll, McCormack, and O'Connor (1976) have suggested.

\section{IRON HYDRIDE IN COOL STARS}

\section{a) Spectroscopic Observations}

Spectrograms of late-type stars were obtained by Cohen with the RCA S-1 image tube and the gold spectrograph mounted on the $2.1 \mathrm{~m}$ telescope at KPNO on 1976 September 12 and 13. The improvised camera was a Nye lens, as owing to back focaldistance problems none of the other gold-spectrograph cameras could be used. The 831 line $\mathrm{mm}^{-1}$ grating blazed at $8000 \AA$ was used in the first order, with an RG 695 filter to block the overlapping second order. This gave a dispersion of $86 \AA \mathrm{mm}^{-1}$, and the grating was tilted to $9200 \AA$ so that the spectra recorded through the $40 \mathrm{~mm}$ diameter image tube begin at $7700 \AA$ near the atmospheric A-band and reach out to the limit of the spectral response of the image tube at approximately $10500 \AA$. The image tube is cooled to a temperature of $-45^{\circ} \mathrm{C}$ by alcohol flowing through dry ice, but even at this low temperature the thermal background limits the exposures to $90 \mathrm{~min}$. The projected slit was $35 \mu \mathrm{m}$, and the spectra were recorded on baked IIIa-J plates. Sensitometer
TABLE 1

STARS OBSERVED

\begin{tabular}{|c|c|c|c|}
\hline Star & $\begin{array}{c}\text { Exposure } \\
\text { Time (min) }\end{array}$ & $V$ & Spectral Type \\
\hline $\begin{array}{l}\text { Ross } 248 \ldots \ldots \\
\text { UV Cet } A+B \ldots \\
\text { W Aql. . . . . . } \\
\text { R Cyg. . . . . }\end{array}$ & $\begin{array}{r}45 \\
50 \\
5 \\
5\end{array}$ & $\begin{array}{c}12.3 \\
12.4+12.9 \\
12.0: \\
12.5:\end{array}$ & $\begin{array}{c}\text { M5e } \\
\text { M6-e } \\
\text { S3,9e-S6,9e } \\
\text { S2.5,9e-S6,8e }\end{array}$ \\
\hline
\end{tabular}

exposures were taken and developed simultaneously with the stellar spectrograms.

The stars observed were $\mathbf{M}$ giants, $\mathbf{M}$ dwarfs, and two S stars. The ones to be discussed here are listed in Table 1. For the $\mathbf{M}$ dwarfs, the visual magnitudes are from Gliese (1969) and the spectral types are from Boeshaar (1976). UV Cet is a close binary and both components were included on the spectrograph slit; Ross 248 is Gliese 905 . For the S stars (both Mira variables) the spectral types are from Keenan (1966), except that the spectral range of $W$ Aql has been extended by including the minimum-light classification of Wing (1972); their visual magnitudes were estimated by Wing at the KPNO $1.3 \mathrm{~m}$ telescope where infrared spectrophotometry was being carried out concurrently for another program. The faint magnitude of $\mathrm{R} \mathrm{Cyg}$ is consistent with its phase, 0.38 cycle past maximum, indicated by the AAVSO predictions for 1976 (Mattei 1976). On the other hand W Aql was predicted to be at phase 0.15 and was expected to be brighter. There $S$ stars are considerably redder than the $\mathbf{M}$ dwarfs, and despite the shorter exposure times used their spectra were somewhat overexposed.

The resolution of the S-1 image tube is approximately $40 \mu \mathrm{m}$; however, the Nye lens (used as the spectrograph camera) was not sturdily mounted and its resolution is not very good. The overall resolution of the spectra is probably closer to $50 \mu \mathrm{m}$ or $4 \AA$.

\section{b) Similarity of Band Structure in $M$ Dwarfs and S Stars}

The plates were traced on the PDS digital microphotometer at KPNO, converted into intensities, and plotted on the CalComp plotter with standard KPNO computer programs. To compensate for the rapidly decreasing response of the S-1 cathode near $10000 \AA$ and for the differences in the colors of $M$ dwarfs and $\mathrm{S}$ stars, each spectrum was multiplied by a linear function of wavelength so that the continuum in the $9800-10200 \AA$ region would be roughly horizontal; this procedure makes it easier to compare the shapes of absorption features in the different stars. Trials with different choices of the continuum line showed that the profile of the $\mathrm{FeH}$ band is quite insensitive to the adopted continuum slope.

Figure 3 shows normalized tracings of the spectra of the stars of Table 1 in the region 9800-10200 $\AA$. The uppermost curve, for comparison, is a degraded (and inverted) laboratory emission spectrum of $\mathrm{FeH}$ from Nordh, Lindgren, and Wing (1977). The most 


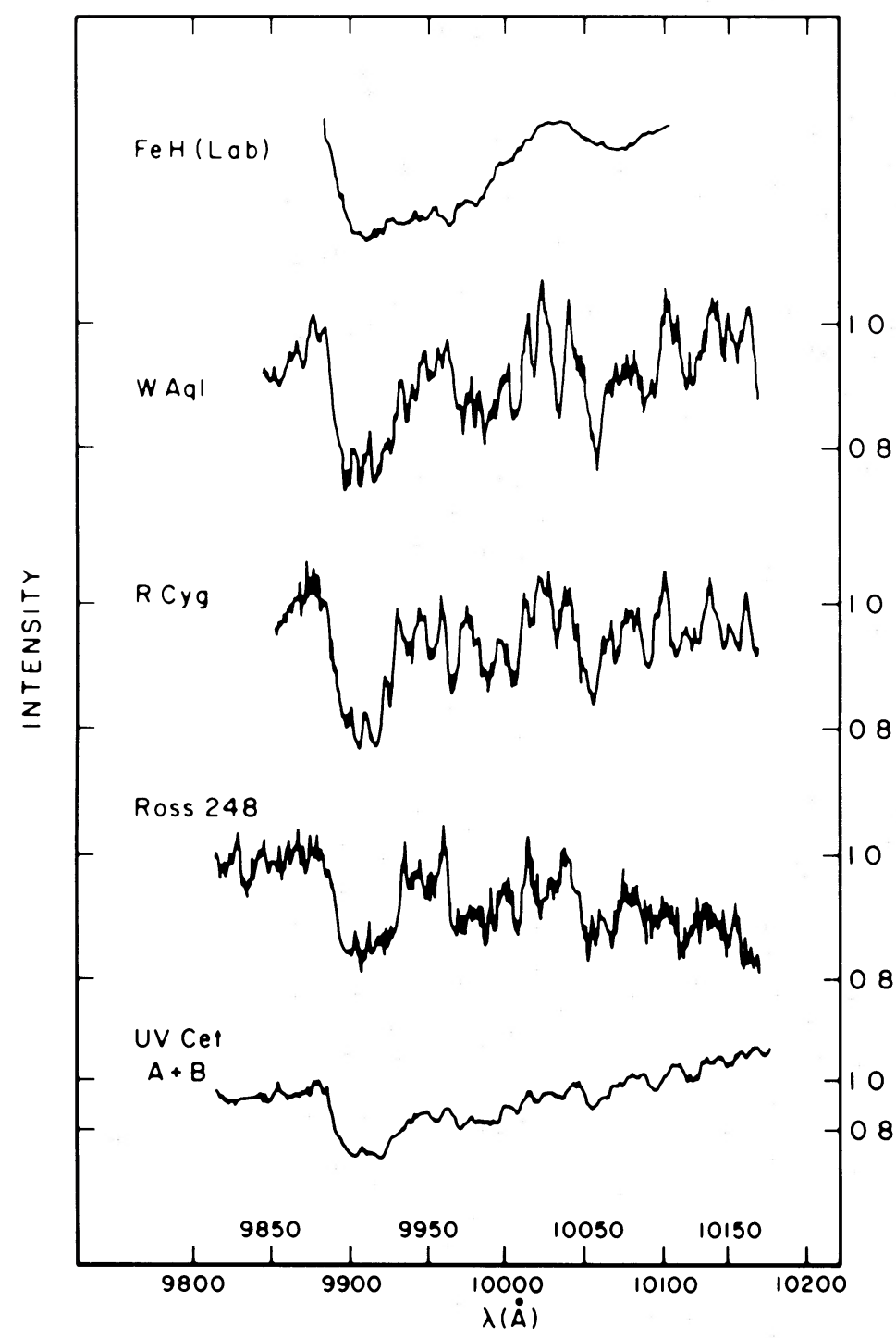

Fig. 3.- Spectra of two S stars (W Aql and R Cyg), two M dwarfs (Ross 248 and UV Cet), and a laboratory source in the region of the $9896 \AA$ band of $\mathrm{FeH}$. The numbers 1.0 and 0.8 at the right specify the intensity scale of each spectrum. The original dispersion of these image-tube plates was $86 \AA \mathrm{mm}^{-1}$.

striking feature of Figure 3 is the close similarity of the absorptions in all four stars. The main absorption feature from about $9900 \AA$ to $9930 \AA$, which is shown at high resolution in the sunspot spectrum of Figure 1 and which in $\mathrm{M}$ dwarfs has been called the Wing-Ford band, has a very similar appearance in the S stars. All four stars also show a second depression from about $9960 \AA$ to $10010 \AA$ and a third region of fairly strong absorption around $10050-10065 \AA$ which is matched by the laboratory spectrum. Further similarities can be found in the finer structure of the spectra. The good agreement of the various spectra leaves no doubt that $\mathrm{FeH}$ is responsible for these absorptions in both $\mathrm{M}$ dwarfs and $\mathrm{S}$ stars.

\section{CONCLUSIONS}

a) Results of this Study

The presence of $\mathrm{FeH}$ molecules in sunspots, indicated earlier by a statistical study of weak lines in the blue and green regions by Carroll, McCormack, and O'Connor (1976), is now firmly established with the identification of at least 332 lines belonging to the infrared bands extending longward from $8691 \AA$ and $9896 \AA$. The absorptions averaged over the $10 \AA$ intervals of greatest line concentration in each band were found to be $7.4 \%$ for the $9896 \AA$ band and $3.7 \%$ for the $8691 \AA$ band. The relative band strengths thus indicated lend support to the suggestion of Carroll, 
McCormack, and O'Connor, based on laboratory deuteride experiments, that the $9896 \AA$ and $8691 \AA$ bands are the $(0,0)$ and $(1,0)$ transitions, respectively.

We were not able to identify the infrared $\mathrm{FeH}$ bands in the spectrum of the solar disk, although several of the strongest $\mathrm{FeH}$ lines fall in stretches of the spectrum that are very clean. This negative result is at variance with the conclusion of Carroll, McCormack, and O'Connor (1976) that "the presence of FeH in both photosphere and spot has been established with a high degree of certainty." Their conclusion was based upon the bands in the blue and green regions-where contamination by other atomic and molecular species is considerable-and upon the fact that the correlations with laboratory $\mathrm{FeH}$ wavelengths were found to be significant for both of the following groups of solar lines: (a) unidentified lines observed in spots only, and (b) unidentified weak lines in the photosphere which are enhanced in spots. While the lines of the latter group undoubtedly owe their enhancements to the presence of $\mathrm{FeH}$ in spots, this does not ensure that $\mathrm{FeH}$ is responsible for the absorption found at these wavelengths in the disk. In view of the negative result given by the infrared bands, we suggest that the only difference between groups $(a)$ and $(b)$ above is that the $\mathrm{FeH}$ lines of one group are unblended, while those of the other group are blended with unidentified atomic or molecular lines that appear weakly in the disk spectrum.

The $9896 \AA$ band has recently been identified by Nordh, Lindgren, and Wing (1977) in the spectra of $M$ dwarfs and stars of type $S$, in each of which it had previously been listed as an unidentified feature. However, all observations of this band in $\mathbf{M}$ dwarfs published to date have had such low resolution that they show only a single absorption feature, about $30-40 \AA$ wide, corresponding to the region of maximum line concentration; for this reason Nordh, Lindgren, and Wing emphasized that observations of higher resolution were needed to confirm their suggestion that the feature has the same origin in $\mathrm{M}$ dwarfs as in $\mathrm{S}$ stars. Our S-1 image-tube spectrograms at $86 \AA \mathrm{mm}^{-1}$ provide this confirmation by showing considerable structure that is virtually identical in both kinds of stars.
Although the Wing-Ford band was used prior to its identification with $\mathrm{FeH}$ as an indicator of the fraction of the light contributed by cool dwarfs to the integrated spectra of external galaxies (see, e.g., Whitford 1977), a knowledge of its identification will increase its usefulness since the band strength will now depend upon metallicity in a known way.

\section{b) Suggestions for Further Work}

We hope that the identification of the infrared bands of $\mathrm{FeH}$ in sunspot spectra will inspire renewed efforts to analyze these bands, since only then will they be useful in quantitative astrophysical investigations. Such an analysis may require deeper, higher-resolution laboratory spectra to record weaker lines and to separate blends. An observation in the $11500 \AA$ region, the expected location of the $(0,1)$ band, would be useful to verify the vibrational assignments proposed by Carroll, McCormack, and O'Connor (1976).

The FTS sunspot spectrum clearly shows the Zeeman patterns of many atomic lines, particularly those of Fe I. Many of the FeH lines are sharp, but others have rather broad, square profiles, perhaps as a result of magnetic splitting. We have not attempted to make use of this information since the bands have not been analyzed. On the other hand, it is possible that a study of the Zeeman patterns of FeH lines may provide the clue needed to unravel the band structure.

It will be important to attempt to measure the strength of some FeH lines in solar disk spectra, weak as they are, since this would permit, among other things, an estimate of the dissociation energy of the $\mathrm{FeH}$ molecule. The lines of the blue and green bands should not be used for this purpose since any absorption in disk spectra at their wavelengths is more likely to be caused by contaminants. We would suggest measuring a few of the strongest lines of the $9896 \AA$ band, where problems with contaminants should be minimal, with the highest possible resolution; specifically, we recommend the groups of lines at 9906-9908 $\AA$ and 9916-9919 $\AA$ (see Fig. 1) and the well-spaced strong lines in the interval 9950-9980 $\mathrm{A}$.

\section{REFERENCES}

Boeshaar, P. C. 1976, Ph.D. thesis, Ohio State University.

Carroll, P. K., and McCormack, P. 1972, Ap. J. (Letters), 177, L33.

Carroll, P. K., McCormack, P., and O'Connor, S. 1976, Ap. J., 208, 903.

Delbouille, L., and Roland, G. 1963, Mém. Soc. Roy. Sci. Liège, Special vol. 4.

Gliese, W. 1969, Veröff. Astr. Rechen-Instituts, Heidelberg, No. 22.

Keenan, P. C. 1966, Ap. J. Suppl. (No. 118), 13, 333.

Klynning, L., and Lindgren, B. 1973, Univ. Stockholm Institute of Physics, Rept. 73-20.

Mattei, J. A. 1976, AAVSO Bull. 39.

McCormack, P., and O'Connor, S. 1976, Astr. Ap. Suppl., 26, 373.

Nordh, H. L., Lindgren, B., and Wing, R. F. 1977, Astr. Ap., 56, 1 .

Whitford, A. E. 1977, Ap. J., 211, 527.

Wing, R. F. 1972, Mém. Soc. Roy. Sci. Liège, 6th ser., 3, 123. Wing, R. F., and Ford, W. K., Jr. 1969, Pub. A.S.P., 81, 527.

J. W. Brault and J. Cohen: Kitt Peak National Observatory, P.O. Box 26732, Tucson, AZ 85726

R. F. WING: Astronomy Department, Ohio State University, 174 W. 18th Ave., Columbus, OH 43210 